Revue d'histoire de l'Amérique française

REVUE D.HISTOIRE DE L'AMÉRIQUE FRANÇAISE

\title{
Une histoire des hommes sans les hommes?
}

\section{Léon Pouliot}

Volume 11, numéro 1, juin 1957

URI : https://id.erudit.org/iderudit/301808ar

DOI : https://doi.org/10.7202/301808ar

Aller au sommaire du numéro

Éditeur(s)

Institut d'histoire de l'Amérique française

ISSN

0035-2357 (imprimé)

1492-1383 (numérique)

Découvrir la revue

\section{Citer ce document}

Pouliot, L. (1957). Une histoire des hommes sans les hommes ? Revue d'histoire de l'Amérique française, 11(1), 110-112. https://doi.org/10.7202/301808ar d'utilisation que vous pouvez consulter en ligne.

https://apropos.erudit.org/fr/usagers/politique-dutilisation/ 


\section{(A TITRE DOCUMENTAIRE) \\ UNE HISTOIRE DES HOMMES SANS LES HOMMES? 1}

«L'HISTOIRE n'a pas plus de droit à la majuscule que la physique ou la géographie. Elle n'est pas une déesse. Elle n'est pas le pseudonyme matérialiste de quelque nouvelle Providence, chargée de projeter dans le futur les prophéties d'un doctrinaire ou les rêves conquérants d'une secte internationale. L'histoire est une enquête, un regard en arrière, une vue sur les temps révolus. Son seul domaine - il est immense - est le passé, le passé entier sous toutes ses formes et sous tous ses aspects. Raconter l'histoire exactement, ce serait tout raconter à la fois, et le récit, pour être complet, durerait aussi longtemps que les siècles écoulés. Idée absurde. Ecrire l'histoire, c'est donc résumer, éliminer, supprimer, choisir. Le temps lui-même choisit, en détruisant les monuments et les archives sur lesquels nous pourrions appuyer certitudes et hypothèses. L'histoire choisit, en limitant ses horizons, en isolant l'objet de ses études. Les circonstances choisissent, parce que chaque génération regarde le passé à travers ses propres préoccupations et récrit l'histoire à la lumière de ses propres expériences.

«Est-ce à dire qu'elle n'est qu'incertitude et relativité ? Assurément non. En dépit des changements de goûts et de soucis, nos bibliothèques contiennent des milliers de bons et solides travaux, d'une méthode très sûre et d'une parfaite loyauté. Même

${ }^{4}$ Vie de M. Faillon, prêtre de Saint-Sulpice, par l'auteur de la Vie de M. Mollevaut, Paris (1877), 175 .

1 Pierre Gaxotte, de l'Académie française. LE FIGARO LITTERAIRE. le 24 novembre 1956, no 553, pp. 1-11. (Communication de J.-J. LEFEBVRE). 
si des érudits heureux ont la chance de les compléter en quelques points, les conclusions en restent indiscutables. On les consulte et on les utilise toujours avec le même fruit, parce qu'ils sont l'essence de textes exactement lus et soigneusement critiqués, de dépouillements complets et de confrontations impartiales.

«Il n'en va pas de même des synthèses plus vastes, plus ambitieuses, mais aussi plus périssables. Le talent de l'auteur, son intelligence, sa perspicacité, sa manière de comprendre la vie et les hommes déterminent nécessairement les caractères de son œuvre. Quelles que soient l'honnêteté et les scrupules de l'historien, il n'y a pas d'histoire impersonnelle. Même dans le raisonnement mathématique, dans la façon plus ou moins élégante, plus ou moins rapide, de mener à bien une démonstration difficile, la personnalité du savant se décèle. Avec mille fois plus de raisons, il en va de même en histoire - et bien davantage. Il existe done de bons historiens et de mauvais, des historiens qui sont très proches de la vérité, qui la saisissent parfois, souvent, d'autres que la passion, le parti pris inconscient, les infirmités de leur être condamnent presque toujours à l'arbitraire et à l'erreur. Il en est, comme Michelet, qui mêlent les traits de lumière aux plus incroyables divagations.

"Placés en face d'une réalité complexe, mouvante, qui ne leur apparaît que par échappées, les historiens ne se résignent pas volontiers à n'y voir que le tableau des passions humaines, des grandeurs, des folies, des vertus et des sottises.

«Si l'expérimentation donne à l'acquis scientifique son caractère de constatations et de lois aisément vérifiables, les historiens ne peuvent, eux, recommencer en laboratoire la bataille des Thermopyles ou la révolution de 1789 pour savoir exactement comment les choses se sont passées, quels éléments et quelles doses d'éléments ont produit la victoire ou l'explosion. Un peu humiliés, souffrant de l'incertitude, ils ont cherché avec entêtement les constantes, les lignes de force permanentes, les enchâ̂nements de faits dominateurs et indépendants qui introduiraient dans leur récit la rectitude et la nécessité par lesquelles leur discipline, cessant d'être seulement narrative et explicative, deviendrait enfin une histoire naturelle, mieux encore une physique des sociétés. Il y a trente ans, les institutions étaient fort à la mode. Elles apparaissaient comme le fil solide auquel s'accrochaient les événements. Ensuite, la lutte de classes, l'évolution économique et l'outillage industriel ont tenu la vedette. Enfin, M. Lucien Febvre inventa l'histoire non-événementielle et une école fort active adopta la formule avec enthousiasme.

«A vrai dire, si l'on comprend bien que l'histoire non- 
événementielle fera fi des événements et des hommes, on ne voit pas très exactement ce qu'elle apporte en échange. Elle décrira les «structures sociales », les «états de civilisation». Sans doute. Montesquieu et Voltaire y avaient pensé avant elle. Elle dira que ces structures et ces états sont changeants, variables et ne se reproduisent jamais exactement. Cette idée est très vieille. Est-ce donc cela l'histoire non-événementielle ?

«Elle consisterait donc à retrancher du passé, à traiter en élément négligeable tout ce qu'on a longtemps considéré comme la matière même de l'historien: la politique, la diplomatie, la guerre, les changements de l'opinion publique, la vie des idées ... Est-ce admissible ? Je suis sûr que l'histoire s'est enrichie, en s'intéressant à la vie des métiers, aux mouvements de la propriété, à l'aspect économique des choses, aux révolutions monétaires, mais je suis également certain qu'elle s'appauvrit, se trahit et fausse le passé en niant tout ce qu'elle méconnaît par système.

«Puisque les structures sociales sont changeantes, pourquoi changent-elles sinon sous le coup d'événements qui peuvent tenir à des découvertes scientifiques, mais aussi, et plus soudainement encore, à des guerres, à des invasions, à des ruptures d'équilibre politique ?

«Est-il possible même d'isoler les facteurs économiques et de les traiter comme s'ils formaient une nécessité indépendante ? Assurément non. Si au XVII ${ }^{e}$ siècle, le mercantilisme correspond bien à un certain état de pénurie monétaire, il n'en est pas moins vrai que Colbert lui a donné en France une forme toute particulière. $\mathrm{Au} \mathrm{XVIII}^{\mathrm{e}}$, des progrès semblables dans la technique agricole ont abouti en Angleterre et en France à des résultats exactement opposés; en Angleterre, la petite propriété paysanne a disparu; en France, elle s'est étendue et consolidée. C'est que dans les deux pays les facteurs économiques ont subi l'influence de facteurs politiques différents.

«La matière de l'histoire est infiniment riche et nous ne devons pas nous insurger contre cette richesse. Tout compte. La machine à vapeur a son importance, qui est immense, mais la clairvoyance d'un chef d'Etat, les talents d'un général en chef peuvent, en de certaines occasions, arrêter, précipiter ou retourner la marche d'une évolution qui semblait fatale. Faire l'histoire des hommes sans les hommes me paraît une gageure impossible à tenir. Il est permis, pour étudier une époque ou une question, de se placer à différents observatoires. Mais quand on est installé dans le sien, il est défendu de nier l'existence de ce qu'on ne voit pas. A plus forte raison de ce qu'on ne veut pas voir... 